\title{
Effect of 6-months of physical exercise on the nitrate/nitrite levels in hypertensive postmenopausal women Pedro R Zaros ${ }^{\dagger 1}$, Carla EM Romero Pires ${ }^{\dagger 1}$, Mauricio Bacci Jr ${ }^{\dagger 2}$, Camila Moraes $^{\dagger 3}$ and Angelina Zanesco* ${ }^{* \dagger 1}$
}

Address: ${ }^{1}$ Department of Physical Education, Institute of Biosciences, University of Sao Paulo State (UNESP), Rio Claro (SP), Brazil, ${ }^{2}$ Departmento of Biochemistry, University of Sao Paulo State (UNESP), Rio Claro (SP), Brazil and ${ }^{3}$ Institute of Physical Activity and Sports, Cruzeiro do Sul University, Sao Paulo (SP) Brazil

Email: Pedro R Zaros - przaros@uol.com.br; Carla EM Romero Pires - carlapires@hotmail.com; Mauricio Bacci - mbacci@rc.unesp.br; Camila Moraes - mormila@hotmail.com; Angelina Zanesco* - azanesco@rc.unesp.br

* Corresponding author †Equal contributors

Published: 19 June 2009

BMC Women's Health 2009, 9:17 doi:10.1186/1472-6874-9-17
Received: 14 November 2008

Accepted: 19 June 2009

This article is available from: http://www.biomedcentral.com/1472-6874/9/17

(C) 2009 Zaros et al; licensee BioMed Central Ltd.

This is an Open Access article distributed under the terms of the Creative Commons Attribution License (http://creativecommons.org/licenses/by/2.0), which permits unrestricted use, distribution, and reproduction in any medium, provided the original work is properly cited.

\begin{abstract}
Background: Evidences have showed that the incidence of arterial hypertension is greater in postmenopausal women as compared to premenopausal. Physical inactivity has been implicated as a major contributor to weight gain and abdominal obesity in postmenopausal women and the incidence of cardiovascular disease increases dramatically after menopause. Additionally, more women than men die each year of coronary heart disease and are twice as likely as men to die within the first year after a heart attack. A healthy lifestyle has been strongly associated with the regular physical activity and evidences have shown that physically active subjects have more longevity with reduction of morbidity and mortality. Nitric oxide (NO) produced by endothelial cells has been implicated in this beneficial effect with improvement of vascular relaxing and reduction in blood pressure in both laboratory animals and human. Although the effect of exercise training in the human cardiovascular system has been largely studied, the majority of these studies were predominantly conducted in men or young volunteers. Therefore, the aim of this work was to investigate the effects of 6 months of dynamic exercise training (ET) on blood pressure and plasma nitrate/nitrite concentration (NOx) in hypertensive postmenopausal women.
\end{abstract}

Methods: Eleven volunteers were submitted to the ET consisting in 3 days a week, each session of 60 minutes during 6 months at moderate intensity (50\% of heart rate reserve). Anthropometric parameters, blood pressure, NOx- concentration were measured at initial time and after ET.

Results: A significant reduction in both systolic and diastolic blood pressure values was seen after ET which was accompanied by markedly increase of NOx- levels (basal: $10 \pm 0.9$; ET: $16 \pm 2 \mu \mathrm{M}$ ). Total cholesterol was significantly reduced (basal: $220 \pm 38$ and ET: $178 \pm 22 \mathrm{mg} / \mathrm{dl}$ ), whereas triglycerides levels were not modified after ET (basal: $141 \pm 89$ and ET: $147 \pm 8 \mathrm{mg} / \mathrm{dl}$ ).

Conclusion: Our study shows that changing in lifestyle promotes reduction of arterial pressure which was accompanied by increase in nitrite/nitrate concentration. Therefore, 6-months of exercise training are an important approach in management arterial hypertension and play a protective effect in postmenopausal women. 


\section{Background}

Epidemiological studies have shown that women life span is eight-year more compared to men. However, the incidence of cardiovascular disease in women increases dramatically after postmenopausal years, for instance, more women than men die each year of coronary heart disease (CHD) and are twice as likely as men to die within the first year after a heart attack. The risk factors of CHD are mainly related to dyslipidemia, arterial hypertension, physical inactivity, overweight, and diabetes mellitus [1]. Additionally, older women are at greater risk for weight gain and abdominal fat accumulation, a major component of metabolic syndrome. Physical inactivity has been implicated as a major contributor to overall weight gain and abdominal obesity in postmenopausal women $[2,3]$.

A healthy lifestyle has been strongly associated with the regular physical activity. Evidences have shown that physically active subjects have more longevity with reduction of morbidity and mortality. Physical exercise prevents or reduces the deleterious effects of pathological conditions such as arterial hypertension, CHD, atherosclerosis, diabetes mellitus, and metabolic syndrome [4].

Nitric oxide (NO) produced by endothelial cells is the major regulator of the cardiovascular system acting on vascular basal tone, inhibiting platelet aggregation and smooth muscle proliferation [5]. The production of NO is regulated by an enzyme named NO synthase (NOS) which is activated by humoral (hormones, autacoids) and physical stimuli (shear stress) [6]. Vascular shear stress is a result of increased blood flow in the vessel wall and it is described as a potent stimulator of NO production from endothelium $[7,8]$. Physical exercise is a powerful stimulus to increase blood flow in vascular beds and consequently the beneficial effects of physical training on cardiovascular diseases are strongly associated with increase in NO production and/or its bioavailability either in human or laboratory animals [9-12]. Regarding the role of NO and physical training, the majority of the studies was predominantly conducted in men or young volunteers, but the effect of long-term of exercise training on the NO production and/or its bioavailability in postmenopausal women has not been investigated. Furthermore, evidences showed that the incidence of arterial hypertension is greater in postmenopausal women as compared to premenopausal age [11]. Therefore, the aim of this work was to investigate the effects of 6 months of dynamic exercise training on blood pressure and plasma nitrate/nitrite concentration in hypertensive postmenopausal women.

\section{Methods}

\section{Participants screening}

This study was approved by the Ethical Committee of the Institute of Bioscience from University of São Paulo State
(UNESP/Rio Claro). The volunteers were recruited by advertisement in the Campus of UNESP and surrounding area. To be included in the study, postmenopausal women must be sedentary (regular aerobic exercise $\leq 2$ sessions/wk and $<20 \mathrm{~min} /$ session, sedentary occupation); non-smoking; no diabetic (fasting glucose level $<110 \mathrm{mg}$ / $\mathrm{dl})$; an average systolic blood pressure from the two screening visits of 140-159 and a diastolic blood pressure of 90-99 mmHg (Stage 1 hypertension); and a medical condition that not exclude the exercise training. The exclusion criteria were subjects with cardiovascular disease (angina, valvular disease, stroke), diabetes, arthritis, alcohol consumption $>3$ drinks per day, orthopedic conditions that affect the ability to participate in exercise program, neurological or psychiatric diseases, and pregnancy. To detect cardiovascular, pulmonary or other disease the volunteers were examined by a physician to selected the populations before the training test. A medical history, exercise training history and dietary history questionnaires were applied to the participants before the inclusion in this study. Eligible subjects were informed of the procedures and risks of the study and signed a written informed consent in accordance with the policies and ethical Committee of the Institute of Bioscience from UNESP. Eleven volunteers were included in this study.

\section{Anthropometric and cardiovascular parameters}

Measurements of height were made using a clinical stadiometer in bare and body weight was measured with a digital calibrated precision scale (Thinner MS-7400, Fairlfiled NJ, USA). Body mass index (BMI) was determined by weight $(\mathrm{Kg})$ divided by the square of the height in meters. Waist-hip ratio values (WHR) were also calculated. The anthropometric measurements were carried out at initial time and after 24 weeks of exercise training.

The volunteers were instructed not to exercise outside of the laboratory before blood pressure measurement. After 15 minutes of seated quiet rest, blood pressure was measured with aneroid sphygmomanometer (Tycos, Raleigh, $\mathrm{NC}$ ) in 2 sessions. Resting heart rate was measured after 5 minutes in orthostatic position using a heart rate monitor (Polar A3, Kempele, Finland).

\section{Total cholesterol, triglycerides and glucose concentration} After 6 months of training exercise, venous blood samples were collected after 12 hours overnight fasting. Plasma was immediately separated by centrifugation $(8,000 \mathrm{~g})$ and Total cholesterol, triglycerides and glucose concentration were measured using specific kits (colorimetric method, Laborlab, São Paulo-SP, Brazil).

\section{Determination of plasma nitrite/nitrate $\left(\mathrm{NO}_{x}{ }^{-}\right)$levels}

In order to evaluate the $\mathrm{NO}$ production, the plasma levels of nitrite and nitrate $\left(\mathrm{NO}_{2}{ }^{-} / \mathrm{NO}_{3}{ }^{-}\right)$were measured. Briefly, immediately after arterial blood collecting, the samples 
were centrifuged $(8,000 \mathrm{~g})$ for $10 \mathrm{~min}$, and the resulting plasma supernatant was stored at $-80^{\circ} \mathrm{C}$. Plasma samples were ultrafiltered through microfilter cups (Microcon Centrifugal Filter Units, $10 \mathrm{kDa}$; Millipore, Bedford, MA, USA). The $\mathrm{NO}_{\mathrm{x}}$ - concentration of the resulting filtrate solution was determined using a commercially available kit (Cayman Chemical, Ann Arbor, MI, USA) according to the manufacturer's instructions. This assay determines the total NO based on the enzymatic conversion of nitrate to nitrite by nitrate reductase. After the conversion, the spectrophotometric measurement of nitrite is accomplished by using the Griess Reaction. The resulting deep purple azo compound absorbs light at 540-550 nm.

\section{Exercise training}

Each exercise session started and ended with appropriate warm-up and cool-down activities. To get familiarized to the cycle ergometer, in the first day of training, the participants performed an initial training session for $20 \mathrm{~min}$ increasing $10 \mathrm{~min}$ a day until 60 minutes of exercise training. The exercise sessions were supervised by personal trainer in all studied time. The participants were submitted to an aerobic exercise program on a cycle ergometer (Moviment, Jau SP, Brazil) in a quiet room with temperature at $25^{\circ} \mathrm{C}$. Exercise sessions were performed for 3 days a week, 60 minutes each session, during 24 weeks with an intensity of $50 \%$ of heart rate reserve, according to the following equation [13].

[(maximal heart rate - resting heart rate $) \times 0.5+$ resting heart rate $]$

\section{Statistics}

Data were analyzed by using GraphPad Instat version 3.0 (GraphPad, San Diego CA, USA) using paired student $t$ test. A $p$ value $<0.05$ was considered as statistically significant.

\section{Results}

Neither BMI (baseline: $33 \pm 1.4$ and ET: $31 \pm 1.5 \mathrm{Kg} \mathrm{m}^{2-1}$ ) nor WHR (baseline: $0.88 \pm 0.06$ and ET: $0.86 \pm 0.05$ ) were altered by exercise training program for 24 weeks. On the other hand, a significant reduction in both systolic (12\%) and diastolic (10\%) blood pressure values was seen after aerobic exercise training in hypertensive postmenopausal women. Heart rate was also reduced (approximately $8 \%$ ) by physical training in this population. Data are summarized in Table 1.

Total cholesterol concentration was significantly reduced after ET, approximately $20 \%$. On the other hand, no changes were seen in the glucose and triglycerides levels. Plasma nitrite/nitrate concentration was markedly increased by 6 -months of dynamic exercise training at moderate intensity in hypertensive postmenopausal
Table I: Anthropometric and cardiovascular parameters at initial time and after 6 months of aerobic exercise in hypertensive postmenopausal women

\begin{tabular}{lcc}
\hline Parameters & Initial time & After 24 weeks \\
\hline Age (years) & $50 \pm 4$ & $50 \pm 4$ \\
Height (cm) & $161 \pm 0.02$ & $161 \pm 0.02$ \\
Weight (kg) & $86 \pm 3.5$ & $82 \pm 3.3$ \\
BMI (kg/m2) & $33 \pm 6$ & $32 \pm 6$ \\
WHR & $0.89 \pm 0.06$ & $0.87 \pm 0.05$ \\
SBP (mmHg) & $141 \pm 10$ & $123 \pm 8^{*}$ \\
DBP (mmHg) & $90 \pm 5$ & $80 \pm 5^{*}$ \\
HR (beats/min) & $83 \pm 5$ & $76 \pm 6^{*}$ \\
\hline
\end{tabular}

Data are mean \pm S.E.M.

BMI: body mass index, WHR: waist-to-hip ratio, SBP: systolic blood pressures, DBP: diastolic blood pressure and HR: heart rate.

$* p<0.05$, statistically different from initial time.

women, approximately 60\%. Data are summarized in Table 2.

\section{Discussion}

Our study shows that exercise training for 6 months promotes reduction in arterial blood pressure that is accompanied with increase in nitrite/nitrate concentrations in hypertensive postmenopausal women.

Physical exercise failed to provoke any alterations in BMI or waist-to-hip ratio in this particular population. The reason for that could be all the volunteers had no caloric intake restriction. Indeed, evidences show that effective loss of body weight is observed mainly when exercise training is associated with dietary restriction $[14,15]$. Another possibility to explain the lack of changing in BMI of the volunteers could be due to the increment of muscle mass in response to physical exercise training. Indeed, previous study showed that body fat percentage is decreased in trained women whereas the lean body mass is increased [16].

It is well established that exercise training promotes beneficial effect on cardiovascular system which has been associated with an improvement of the NO-cGMP pathway in the vasculature in both human and laboratory ani-

Table 2: Lipid profile and glucose concentration at initial time and after 6 months of aerobic exercise in hypertensive postmenopausal women

\begin{tabular}{lcc}
\hline Parameters & Initial time & 24 weeks \\
\hline Total cholesterol(mg/dl) & $220 \pm 38$ & $178 \pm 22^{*}$ \\
Triglycerides $(\mathrm{mg} / \mathrm{dl})$ & $141 \pm 89$ & $147 \pm 88$ \\
Glucose $(\mathrm{mg} / \mathrm{dl})$ & $99 \pm 16$ & $93 \pm 16$ \\
Nitrite/Nitrate $(\mathrm{mol} / \mathrm{L})$ & $10 \pm 0.9$ & $16 \pm 2^{*}$ \\
\hline
\end{tabular}

Data are means \pm S.E.M for II volunteers.

*statistically different from initial time. 
mals. Thus, exercise training is an important nonpharmacological tool in management arterial hypertension and atherosclerotic process $[9,10]$. Evidences have showed that the incidence of arterial hypertension is greater in postmenopausal women as compared to premenopausal. A number of factors can contribute to this increase in incidence of arterial hypertension in postmenopausal women including estrogen deficiency, alterations in lipid profile, weight gain, and decreases in physical activity during the menopause $[2,3,17,18]$. Indeed, a recent study has shown that estradiol deficiency tends to have a negative influence on NO-cGMP pathway in menopausal women, thus modification in lifestyle by physical exercise is an important approach to prevent cardiovascular complications in this population [19]. Our study shows that 6-month of dynamic exercise training was effective in lowering both systolic and diastolic blood pressure in a similar manner. This reduction was positively associated with increase in nitrite/nitrate concentration which reflects NO production. Considering that preventive actions to avoid high incidence of cardiovascular disease mainly arterial hypertension are extremely necessary for postmenopausal women, our results reinforce previous studies [20,21] and extend these findings showing that 6-months of exercise training (24 weeks) promotes a considerable increase in plasma nitrite/nitrate concentration (approximately 60\%) as compared to studies where the physical exercise was applied for only 12 weeks with increment of $15-35 \%$ in plasma nitrite/nitrate concentration [20,21]. Thus, our findings show the improvement in cardiovascular system regulation is timedependent of exercise training for this population.

Alterations in lipid profile play an important role for development of atherosclerosis and physical exercise has been successful in management lipid concentration in elderly volunteers [22]. In our studied population, the concentration of total cholesterol was slightly elevated according to the National Cholesterol Education Program's clinical practice guideline $(>200 \mathrm{mg} / \mathrm{dl})$, whereas triglycerides level was at normal range $(<150 \mathrm{mg} / \mathrm{dl})$. Aerobic physical training for 24 weeks provokes a significant decrease in total cholesterol concentrations in hypertensive postmenopausal women, but no changes in triglycerides levels were observed. As expected, physical exercise was effective to modify the elevated concentration of total cholesterol, but not triglycerides levels which is in agreement of the literature showing that alterations is observed when exist a previous abnormality.

\section{Conclusion}

Our study shows that changing in lifestyle promotes reduction of arterial pressure which was accompanied by a marked increase in nitrite/nitrate concentration. Therefore, 6-months of exercise training are an important approach in management cardiovascular disease risk factors and play a protective effect in atherosclerosis in this particular population.

\section{Competing interests}

The authors declare that they have no competing interests.

\section{Authors' contributions}

PRZ carried out the training program studies, participated in the sequence alignment and drafted the manuscript. CEMRP helped carried out training program studies. MBJr helped with nitrite/nitrate analysis. $\mathrm{CM}$ in the design of the study and performed the statistical analysis. AZ conceived of the study, and participated in its design and coordination and helped to draft the manuscript. All authors read and approved the final manuscript.

\section{Acknowledgements}

The authors are grateful to Fundação de Amparo a Pesquisa do Estado de Sao Paulo (FAPESP) for financial support.

\section{References}

I. Harrison-Bernard LM, Raij L: Postmenopausal hypertension. Curr Hypertens Rep 2000, 2:202-207.

2. Simkin-Silverman L, Wing RR, Hansen DH, Klem ML, PasagianMacaulay AP, Meilahn EN, Kuller LH: Prevention of cardiovascular risk factor elevations in healthy premenopausal women. Preventive Medicine 1995, 24:509-5I7.

3. Lewis SJ, Whalen EJ, Beltz TG, Johnson AK: Role of the anterior region of the third ventricle in the cardiovascular responses produced by systemic injection of a nitric oxide synthase inhibitor. Brain Res 1999, 830:191-194.

4. Zanesco A, Antunes E: Effects of exercise training on the cardiovascular system: Phamacological approaches. Pharmacol Ther 2007, I | 4:307-3 I7.

5. Murad F: Cellular signaling with nitric oxide and cyclic GMP. Braz J Med Biol Res 1999, 32(I I): I317-I327.

6. Vanhoutte PM: Endothelial control of vasomotor function. Cir Res 2003, 67:572-575.

7. Traub $O$, Berk BC: Laminar shear stress mechanisms by which endothelial cells transduce an atheroprotective force. Arterioscler Thromb Vasc Biol 1998, 18:677-685.

8. Boo YC, Jo H: Flow-dependent regulation of endothelial nitric oxide synthase: role of protein kinases. Am J Physiol Cell Physiol 2003, 285(3): C499-C508.

9. Kingwell BA: Nitric oxide-mediated metabolic regulation during exercise: effects of training in health and cardiovascular disease. FASEB JOURNAL 2000, I4:1685-1696.

10. Grahan DA, Rush JW: Exercise training improves aortic endothelium-dependent vasorelaxation and determinants of nitric oxide bioavailabity in spontaneously hypertensive rats. Journal Appl Physiol 2004, 96:2088-2096.

II. Higashi $Y$, Yoshizumi M: Exercise and endothelial function: role of endothelium-derived nitric oxide and oxidative stress in healthy subjects and hypertensive patients. Pharmacol Ther 2004, 102:87-96.

12. de Moraes C, Davel AP, Rossoni LV, Antunes E, Zanesco A: Exercise training improves relaxation response and SOD-I expression in aortic and mesenteric rings from high caloric diet-fed rats. BMC Physiol 2008, 8: 12.

13. ACSM: Position stand on the recommended quantaty and quality of exercise for developing and maintaining cardiorespiratory and muscular fitness, and flexibility in adults. Med Sci Sports Exer 1998, 30:975-991.

14. Shaw K, Gennat H, O'Rourke P, Del Mar C: Exercise for overweight or obesity. Cochrane Database Syst Rev 2006, 04:CD0038I7.

15. Hansen KC, Shang Z, Gomez T, Adams AK, Schoeller DA: Exercise increases the proportions of fat utilization during short term 
consumption of a high fat diet. Am Journal Clin Nut 2007, 85:109-II6.

16. Staron RS, Malicky ES, Leonardi MJ, Falkel JE, Hagerman FC, Dudley GA: Muscle hypertrophy and fast fiber type conversions in heavy resistance-trained women. Eur Journal Appl Physiol Occup Physiol 1990, 60:71-79.

17. Speroff L, Lobo RA: Postomenopausal hormone therapy and the cardiovascular system. Heart Dis Stroke 1994, 03:173-176.

18. Anderson WF, Chatterjee N, Ershler WB, Brawley OW: Estrogen receptor breast cancer phenotypes in the Surveillance, Epidemiology and End Results database. Breast Cancer Res Treat 2002, 01:27-36.

19. Salhotra S, Arora S, Trived SS, Bhattacharjee J: Influence of menopause on biochemical markers of endothelial dysfunction-A case-control pilot study in North Indian population. Maturitas 2009, 62:166-170.

20. Maeda S, Tanabe T, Otsuki T, Suguwara J, lemitsu M, Miyauchi T, Kuno S, Ajisaka R, Matsuda M: Moderate regular exercise increases basal production of nitric oxide in elderly women. Hypertension Res 2004, 27:947-953.

21. Ohta M, Nanri H, Matsushima Y, Sato Y, lkeda M: Blood pressurelowering effects of lifestyle modification: possible involvement of nitric oxide bioavailability. Hypertens Res 2005, 10:779-786.

22. Halverstadt A, Phares DA, Wilund KR, Goldberg AP, Hagberg JM: Endurance exercise training raises high-density lipoprotein cholesterol and lowers small low-density lipoprotein and very low-density lipoprotein independent of body fat phenotypes in older men and women. Metabolism 2007, 56:444-450.

\section{Pre-publication history}

The pre-publication history for this paper can be accessed here:

http://www.biomedcentral.com/1472-6874/9/17/prepub
Publish with Biomed Central and every scientist can read your work free of charge

"BioMed Central will be the most significant development for disseminating the results of biomedical research in our lifetime. "

Sir Paul Nurse, Cancer Research UK

Your research papers will be:

- available free of charge to the entire biomedical community

- peer reviewed and published immediately upon acceptance

- cited in PubMed and archived on PubMed Central

- yours - you keep the copyright 